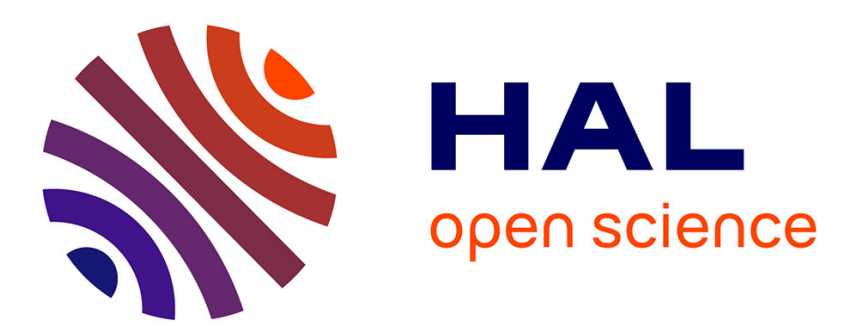

\title{
A Fatty Acid Anabolic Pathway in Specialized-Cells Remotely Controls Oocyte Activation in Drosophila
} Mickael Poidevin, Nicolas Mazuras, Gwénaëlle Bontonou, Pierre Delamotte, Béatrice Denis, Maëlle Devilliers, Delphine Petit, Claude Wicker-Thomas, Jacques Montagne

\section{To cite this version:}

Mickael Poidevin, Nicolas Mazuras, Gwénaëlle Bontonou, Pierre Delamotte, Béatrice Denis, et al.. A Fatty Acid Anabolic Pathway in Specialized-Cells Remotely Controls Oocyte Activation in Drosophila. 2021. hal-03455477

\author{
HAL Id: hal-03455477 \\ https://hal.science/hal-03455477
}

Preprint submitted on 29 Nov 2021

HAL is a multi-disciplinary open access archive for the deposit and dissemination of scientific research documents, whether they are published or not. The documents may come from teaching and research institutions in France or abroad, or from public or private research centers.
L'archive ouverte pluridisciplinaire HAL, est destinée au dépôt et à la diffusion de documents scientifiques de niveau recherche, publiés ou non, émanant des établissements d'enseignement et de recherche français ou étrangers, des laboratoires publics ou privés. 


\section{A Fatty Acid Anabolic Pathway in Specialized-Cells Remotely}

4 Mickael Poidevin ${ }^{a}$, Nicolas Mazuras ${ }^{\text {b,2 }}$, Gwénaëlle Bontonou ${ }^{\text {b,3 }}$, Pierre Delamotte ${ }^{\text {a }}$, Béatrice

5 Denis $^{b}$, Maëlle Devilliers ${ }^{a}$, Delphine Petit ${ }^{a}$, Claude Wicker-Thomas ${ }^{b, 1}$, Jacques Montagne ${ }^{\mathrm{a}, 1}$

6

a Institut for Integrative Biology of the Cell (I2BC), UMR 9198, CNRS, Université Paris-Sud, CEA, 91190, Gif-sur-Yvette, France

b Laboratoire Evolution, Génomes, Comportements, Ecologie (EGCE), UMR 9191, CNRS, IRD,

10 Université Paris-Sud and Université Paris-Saclay, 91190, Gif-sur-Yvette, France

${ }^{1}$ CWT and JM contributed equally to the work. Correspondence:

cwickerthomas@gmail.com ; Jacques.MONTAGNE@i2bc.paris-saclay.fr

${ }^{3}$ Present address: Department of Ecology and Evolution, UNIL, CH-1015 Lausanne 
24 Pheromone-mediated partner recognition is crucial for maintenance of animal species. Here, we 25 discover a metabolic link between pheromone and gamete physiology. In female genital tract, 26 oocyte maturation is arrested at a specific meiotic-phase. Release of this arrest, called oocyte27 activation, is triggered by a species-dependent signal. We show in Drosophila melanogaster 28 that oenocytes, which produce the fatty acids (FAs) used as precursors of cuticular 29 hydrocarbons (CHCs), including pheromones, are also essential for oocyte activation. We identified a set of FA-anabolic enzymes required within oenocytes for the synthesis of a 31 particular FA that is not a $\mathrm{CHC}$ precursor but controls oocyte activation. Our study thus reveals 32 that two tightly linked FA-anabolic pathways act in parallel, one to produce sexual pheromones, 33 the other to initiate embryonic development. Given that pheromone-deficient Drosophila 34 melanogaster females are highly attractive for males irrespective of their species, this oenocyte function might have evolved to prevent hybrid development. 


\section{INTRODUCTION}

In multicellular organisms, sexual reproduction is crucial for species survival. Meeting mates requires pheromonal signals between individuals of the same species (Rekwot, Ogwu et al., 2001). After mating, sperm remains in the female genital tract, where its survival duration varies amongst species (Neubaum \& Wolfner, 1999). In mammalian females, sperm entry into the oocyte triggers a calcium signal that induces oocyte activation (Kashir, Nomikos et al., 2014, Miao \& Williams, 2012, Swann \& Lai, 2016). The regulation of sexual reproduction integrates several physiological inputs, which are not fully characterized. Given the plethora of genetic tools allowing tissue targeted gene miss-expression, Drosophila melanogaster provides a convenient model system to address these issues.

In Drosophila females, oogenesis produces oocytes arrested at metaphase I of meiosis. Oocyte activation also depends on a calcium signal (Sartain \& Wolfner, 2013), which is not triggered by sperm entry, but proceeds while the oocyte moves through the oviduct and the uterus (Heifetz, Yu et al., 2001). Activation provokes i) meiosis completion, ii) formation of the vitelline membrane that prevents polyspermy and iii) translation of maternally provided mRNAs (AvilesPagan \& Orr-Weaver, 2018, Horner \& Wolfner, 2008b, Krauchunas \& Wolfner, 2013). In well-fed fertilized females, eggs are continuously produced and spermatozoa stored in the seminal receptacle and spermathecae are synchronously delivered to fertilize the oocytes, indicating that regulatory processes coordinate nutritional status with oogenesis, and egg laying with sperm delivery (Avila, Bloch Qazi et al., 2012, Wolfner, 2011).

Drosophila sexual pheromones are cuticular hydrocarbon $(\mathrm{CHC})$ members (Montagne J. \& Wicker-Thomas, 2020) that form by decarboxylation of very long chain fatty acids (VLCFAs) (Qiu, Tittiger et al., 2012). In eukaryotes, VLCFA synthesis is catalyzed by the elongase complex from a LCFA (long chain fatty acid) substrate (Jakobsson, Westerberg et al., 2006), whereas LCFA synthesis is catalyzed by FASN (fatty acid synthase) from an acetyl-CoA primer (Maier, Leibundgut et al., 2010). Synthesis of both LCFAs and VLCFAs requires the sequential incorporation of malonyl-CoA units, whose biogenesis is catalyzed by the Acetyl-CoA 
carboxylase (ACC) (Barber, Price et al., 2005). Regarding Drosophila CHCs, we previously showed that synthesis of VLCFAs takes place exclusively in the oenocytes, which are rows of cells located underneath the dorsal abdominal cuticle, whereas the LCFAs used as precursors are of flexible origin (Wicker-Thomas, Garrido et al., 2015). The Drosophila genome encodes three FASN genes, two of them, FASN2 and FASN3, are specifically expressed in the oenocytes (Chung, Loehlin et al., 2014, Garrido, Rubin et al., 2015, Parvy, Napal et al., 2012). It has been shown that FASN2 catalyzes the synthesis of methylated/branched(mb)FAs, using a primer distinct from acetyl-CoA (Chung et al., 2014, Wicker-Thomas et al., 2015). Regarding FASN3, we previously reported that its knockdown affects tracheal waterproofing in larvae (Parvy et al., 2012) and desiccation resistance but not $\mathrm{CHC}$ synthesis in adult flies (WickerThomas et al., 2015). Here, we show that a particular FA-anabolic pathway operating from the oenocytes remotely controls oocyte activation. Our study thus suggests a metabolic link between pheromone synthesis and female fertility.

\section{RESULTS}

Identification of FA-metabolic genes required in the oenocytes for female fertility

While studying CHCs, we observed that Drosophila females deficient for FASN3 in their oenocytes exhibited a sterile phenotype. To identify additional genes potentially involved in this process, we directed inducible interfering RNA (RNAl) to 57 genes encoding enzymes related to FA-metabolism, using the 1407-gal4 driver that is active in oenocytes from mid L3 larval stage to adulthood (Wicker-Thomas et al., 2015). In their progeny, at least twenty virgin femaleshereafter referred as $1407>$ geneX-RNAi- were individually crossed to Canton-S males. Next, females were transferred every second day to new vials and the number of emerging adults was counted. This way, we identified four additional gene products required for fertility (Table EV1 and Fig EV1A), including ACC, a component of the elongase complex (KAR), a bipartite FAtransporter/acyl-CoA ligase (FATP) and CG6432, which encodes a putative short chain acyl- 
females, yet none of them appeared to be sterile (Fig EV1B). Taken together, these findings reveal that a VLCFA produced in the oenocytes controls female but not male fertility.

\section{Searching for oenocyte defects}

$K A R$, which encodes a component of the elongase complex, has also been shown to prevent oenocyte degeneration in adult flies (Chiang, Tan et al., 2016). We therefore, investigated whether the oenocyte knockdowns that produced female sterility also affected oenocyte viability in females. Consistently, 1407>KAR-RNAi resulted in oenocyte degeneration in 27-day old females, but not in 10- and 18-day old females (Fig EV2A-F). As previously described (WickerThomas et al., 2015), 1407>ACC-RNAi induced lipid accumulation in the oenocytes but did not affect their viability (Fig EV2G-I), whereas 1407>FATP-RNAi resulted in oenocyte degeneration that was visible as of day 18 (Fig EV2J-L). In contrast, 1407>FASN3-RNAi and 1407>CG6432RNAi flies were fully viable and the oenocytes of females did not degenerate, did not accumulate high amounts of lipid droplets and appeared similar to controls (Fig EV2M-R). Following knockdown of any of the genes identified, the female sterile phenotype was invariably observed as of day 8, ie. when oenocytes appeared viable (Fig EV2D,G,J,M,P), indicating that the sterile phenotype resulted from the inactivation of a specific FA metabolic pathway, rather than deficient oenocytes.

\section{Metabolic pathway required for female fertility}

CG6432 is proposed to encode a short chain acyl-CoA ligase (Flybase, 2003). However, protein blast analysis revealed that it contains a putative propionyl-CoA synthase domain and that its closest homologue is a short chain FA-acyl-CoA ligase in mouse (Acss3), and an acetyl-CoA ligase in yeast (Acs1) (Fig EV3). Therefore, we investigated whether CG6432 was also required in other FA-anabolic pathways. We previously reported that oenocyte knockdown of ACC, FASN3, KAR and FATP in young larvae results in flooding of the tracheal system and in lethality 
124 at the L2/L3 transition (Parvy et al., 2012). As previously reported for FASN3 (Garrido et al., 125 2015), this phenotype also happened for oenocyte or ubiquitous knockdown of CG6432 (Fig $1261 \mathrm{~A}$ ), indicating that the CG6432 gene product resides in the oenocyte-specific metabolic 127 pathway that controls tracheal watertightness in larvae. We also previously reported that knockdown of $A C C, K A R$ and FATP, but not of FASN3, in adult oenocytes results in a drop of

129 total $\mathrm{CHC}$ amounts (Wicker-Thomas et al., 2015). Knockdown of CG6432 in adult oenocytes did

130 not reduce total $\mathrm{CHC}$ amounts but resulted in a dramatic drop of 131 methylated/branched(mb)CHCs (Table EV2, Fig 1B and Fig EV4), a phenotype previously 132 reported in FASN2 oenocyte-knockdown flies (Chung et al., 2014, Wicker-Thomas et al., 2015). 133 In contrast to FASN1, fat body knockdown of CG6432 did not reduce total triacylglycerol levels 134 (Fig 1C), indicating that it is not required for FASN1 activity in the fat body. Taken together, 135 these findings reveal that the enzyme encoded by CG6432 selectively resides in the FASN2 136 and FASN3 but not FASN1 anabolic pathway.

137 Next, we made use of the promE-gal4 driver, which, in contrast to the 1407-gal4 driver (Wicker138 Thomas et al., 2015), is active only in the oenocytes in Drosophila females as of stage L1 139 (Billeter, Atallah et al., 2009). All the genes required in adult oenocytes for female fertility (Table 140 EV1) are also essential in larval oenocytes for spiracle watertightness, except the elongase 141 subunit elo ${ }^{C G 6660}$, the knockdown of which seemed to affect only the latter process (Parvy et al., 142 2012). Therefore, to retest elo ${ }^{C G 6660}$ with the promE-gal4 driver we used a different RNAi line 143 (Table EV1). Given that promE-gal4 induced larval lethality when driving any of the genes of 144 interest, its activity was blocked until early metamorphosis using the thermo-sensitive Gal4 145 inhibitor, Gal80 $0^{\text {ts }}$. RNAi expression was induced by a temperature shift to $27^{\circ} \mathrm{C}$. Emerging 146 females were maintained at $27^{\circ} \mathrm{C}$, mated $3-5$ days after virgin collection and changed to new 147 vials every second day (Fig 1D). In this way, newly fertilized females expressing any of the 148 RNAis of interest exhibited a net reduction of fertility compared to that of control females, which 149 quickly dropped to complete sterility (Fig 1D). Moreover, in contrast to the fertility of 150 1407>elo ${ }^{C G 6660}$-RNAi females (Fig EV1A), promE-gal4>elo ${ }^{C G 6660}$-RNAi females appeared to be 
151 sterile, possibly because of different RNAi strength (Fig 1D). Taken together, these findings 152 reveal that a FA anabolic pathway working in the oenocytes produces a particular VLCFA that is 153 required for female fertility (Fig 1E). While the acyl-CoA primer for mbLCFA synthesis catalyzed 154 by FASN2 is likely a propionyl-CoA, whereas the one used by FASN3 is yet unknown. Thus, it is tempting to speculate that CG6432 is responsible for the synthesis of this unconventional FA 156 primer (Fig 1E).

\section{Oenocytes control female fertility}

159 To get further insights into this oenocyte function, we focused on FASN3 and CG6432, since both these genes are essential only in the oenocytes and their knockdown alters neither

161 oenocyte viability (Fig EV2) nor CHC synthesis (Fig 1B and (Wicker-Thomas et al., 2015)).

162 Furthermore, confocal imaging of oenocytes (Fig 2A) and ovaries (Fig 2B) of promE>FASN3-

$163 R N A i$ and promE>CG6432-RNAi females dissected 10 days after mating, revealed no apparent 164 defects, all the egg chamber stages were visible (Fig 2B). Moreover, in mating assays, single 165 wild type males did not exhibit any significant preference when given a choice between a wild 166 type female and a female of either genotype promE>FASN3-RNAi or promE>CG6432-RNAi

167 (Fig 2C). Next, we monitored the number of eggs laid by females. Control, promE $>$ FASN3-RNA $i$ 168 and promE>CG6432-RNAi females laid high amounts of eggs the day after fertilization (Fig 2D); 169 this number decreased the following days, although this effect was more pronounced for 170 promE>FASN3-RNAi and promE>CG6432-RNAi compared to control females (Fig 2D). 171 However, consistent with a fertility defect, the number of eggs that formed pupae in the FASN3172 and CG6432-RNAi conditions dropped more dramatically than the number of eggs laid, as 173 compared to control (Fig 2E-F). Taken together, these findings reveal that the oenocyte 174 metabolic pathway that controls female fertility affects neither partner mating nor oogenesis. 
177 After copulation, spermatozoa are stored for several days within the seminal receptacle and

178 spermathecae of Drosophila females (Wolfner, 2011). We searched for potential defects in 179 sperm storage or delivery. In control females, the number of spermatozoa in the seminal 180 receptacle progressively decreased after mating, while females laid eggs continuously (Fig 3A); promE $>$ FASN3-RNAi and promE>CG6432-RNAi females contained roughly the same number

182 of spermatozoa in their seminal receptacle the day after mating, but surprisingly, this number

183 decreased much less during the following days compared to control females (Fig $3 \mathrm{~A}$ ). Next, we

184 monitored sperm motility in the seminal receptacle but did not observe a difference in sperm speed in promE>FASN3-RNAi and promE>CG6432-RNAi females compared to control females (Fig 3B and movies EV1-3). Moreover, confocal analysis revealed that the eggs of control, promE $>$ FASN3-RNAi and promE>CG6432-RNAi females were fertilized as shown by the presence of sperm flagella (Fig 3C-E). These findings indicate that, despite sperm retention in storage organs, the defect of fertility is not due to a failure of sperm entry into the oocyte.

\section{Oenocytes control oocyte activation}

192 A fertilized egg contains potentially five nuclei, the sperm and oocyte pronuclei, plus three polar

193 globules. However, while analyzing the presence of spermatozoa, we noticed an apparent

194 defect in the number of nuclei in promE>FASN3-RNAi and promE>CG6432-RNAi females (Fig

$1953 \mathrm{C}-\mathrm{E})$. We therefore, counted the number of nuclei in eggs laid by virgin females and observed

196 that the number of nuclei in control eggs varid from zero to four, likely because some nuclei are

197 not stained or not visible (Fig 4A and 4D). Nevertheless, the number of nuclei in promE $>$ FASN3-RNAi and promE $>$ CG6432-RNAi females was much lower than in control

199 females (Fig 4B-C and 4D). Production of the three polar globules results from meiosis 200 completion (Page \& Orr-Weaver, 1997), suggesting that this process does not fully operate in 201 promE>FASN3-RNAi and promE>CG6432-RNAi females. Meiosis completion is triggered by 202 oocyte activation, which also induces formation of the vitelline membrane and translation of 203 maternally provided mRNAs (Horner \& Wolfner, 2008a). The vitelline membrane allows 
204 resistance to bleach induced egg lysis (Horner, Czank et al., 2006). Importantly, we observed 205 that fertilized eggs laid by promE $>F A S N 3-R N A i$ and promE $>C G 6432-R N A i$ females were much 206 less resistant to bleach treatment (Fig 4E). However, the bleach concentration required for egg 207 lysis was higher than the one described by others (Horner et al., 2006). Finally, we analyzed 208 Smaug, a protein encoded by a maternally provided mRNA, whose translation is induced by egg 209 activation (Horner \& Wolfner, 2008a, Tadros, Goldman et al., 2007). Western-blot analysis 210 revealed that Smaug was present at much lower levels in eggs laid by promE $>F A S N 3-R N A i$ 211 and promE>CG6432-RNAi than in eggs laid by control females (Fig 4F). Taken together these 212 findings reveal that a particular VLCFA produced in the oenocytes controls oocyte activation.

\section{DISCUSSION}

215 In this study, we provide evidence that a FA-anabolic pathway, which takes place within the 216 oenocytes of Drosophila females, remotely controls fertility. We have identified six FA-anabolic 217 enzymes required for this process: ACC, FASN3, CG6432, FATP, KAR and Elo ${ }^{\mathrm{CG} 6660}$. ACC, the 218 rate-limiting enzyme for FA synthesis, catalyzes malonyl-CoA synthesis (Barber et al., 2005). 219 FASN3 is one of the three Drosophila FASN enzymes (Garrido et al., 2015). CG6432 that encodes a putative short chain acyl-CoA synthase, might catalyze the synthesis of the acyl-CoA 221 primer used by FASN2 and FASN3 to build up FAs (Fig 1D). FATP is a bipartite fatty acid 222 transporter/acyl-CoA synthase, although our previous study supports that it works instead as an 223 acyl-CoA synthase tightly linked to VLCFA synthesis (Wicker-Thomas et al., 2015). KAR and 224 Elo ${ }^{\mathrm{CG} 6660}$ are subunits of the elongase complex that comprises two reductases (KAR and TER), 225 a dehydratase and the elongase (Jakobsson et al., 2006). The elongase subunit assigns 226 specificity to FA primer usage and to the VLCFA produced; both of which remain unidentified for 227 Elo ${ }^{\mathrm{CG} 6660}$, as for most of the 20 elongases encoded by the Drosophila genome (Montagne J. \& 228 Wicker-Thomas, 2020). Nonetheless, the oenocyte-mediated control of female fertility likely 229 relies on either a single or a restricted subclass of VLCFA(s), which might be the precursor of a 230 yet unknown lipid hormone. We also observed that oenocyte knockdown of FATP and KAR, but 
231 not of ACC, FASN3 and CG6432, results in oenocyte degeneration, further supporting the 232 notion that FATP operates closely to VLCFA synthesis (Wicker-Thomas et al., 2015). 233 Potentially, the degeneration process is not induced by the lack of VLCFAs but by the 234 accumulation of LCFA precursors, which happens when inhibiting VLCFA but not LCFA synthesis. These six enzymes are also required in larval oenocytes to maintain the spiracle 236 watertightness (Parvy et al., 2012), suggesting that FASN3-dependent VLCFAs are the 237 precursors of lipid messengers transmitted through the haemolymph to their target tissues. Our 238 study demonstrates that one of these particular VLCFAs operates on oocyte activation. In 239 females deficient for this VLCFA, i) the low number of nuclei in unfertilized eggs suggests that 240 meiosis completion does not fully operate, ii) the bleach sensitivity of eggs argues for a vitelline 241 membrane defect, and iii) the low levels of Smg suggests a defect in the translation of 242 maternally provided mRNAs. All these processes are typically triggered by oocyte activation 243 (Aviles-Pagan \& Orr-Weaver, 2018). Nonetheless, the lack of this remote control is unlikely to 244 result in a total blockade of oocyte activation, since all these processes are affected but not fully 245 suppressed.

246 At first glance, it is surprising that a VLCFA synthesized through a metabolic pathway tightly 247 parallel to the one responsible for pheromone biogenesis, remotely controls oocyte activation. 248 Of note, it has been shown that Drosophila melanogaster females devoid of oenocytes are 249 attractive to males from other species, including $D$. simulans, $D$. yakuba and $D$. erecta (Billeter 250 et al., 2009). These females are also more attractive to wild type Drosophila melanogaster 251 males, since the delay for copulation with oenocyte deficient females in shortened. The 252 increased attractiveness likely depends on carboxyl-methylated FAs produced by Drosophila 253 females, since shortening of the copulation delay does not happen with males deficient for the 254 odorant receptor of these carboxyl-methylated FAs. These studies support the notion that 255 female attractiveness depends on a balance between repulsion and attraction, where the 256 cocktail of CHC-related compounds would be rather repulsive, while the species distinctive 257 pheromone signature confers the selective attractiveness for conspecific males. Given that 
258 pheromone biogenesis shares common enzymes with the synthesis of the oocyte-activating

259 VLCFA, deficiency in the former pathway should also impede the latter. Therefore, it is tempting

260 to speculate that these two pathways have been co-selected throughout evolution to prevent

261 hybrid development. Flies harboring non-functional oenocytes would copulate with males

262 irrespective of their species with the resulting hybrids being potential competitors. Identification

263 of the FASN3-dependent VLFCAs will constitute the next challenge and should allow

264 deciphering its action mechanism on oocyte activation and determining whether a similar

265 regulatory process is conserved throughout evolution to favor species isolation.

\section{Acknowledgements}

268 We wish to thank Benjamin Loppin for critical advices, VDRC, NIG-FLY M Simonelig for fly 269 stocks and reagents, and Melanie Gettings for manuscript editing. Thanks are due to funding 270 supports from Centre National de la Recherche Scientifique (to CWT, JM), IFR115 grant (to 271 CWT and JM), Fondation ARC (1555286 to JM), French league against Cancer (M27218 to 272 JM), French Government (fellowships MENRT 2015-155 to MD and 2020-110 to PD).

274 Author contributions: CWT and JM conceived the study; MP, NM, PD, BD, MD, DP, CWT and 275 JM performed the methodology; MP, GW, CWT and JM analyzed the data; JM wrote the 276 manuscript. All authors reviewed the manuscript.

278 Competing interest: The authors declare that they have no conflict of interest. 
282 Fly stock: 1407-gal4 (oenocytes from mid L3 stage) (Ferveur, Savarit et al., 1997), BO-gal4 283 (oenocytes in embryo and early larvae) (Gutierrez, Wiggins et al., 2007), promE-gal4 284 (oenocytes from L1 stage) (Billeter et al., 2009), P[w8, ProtB-DsRed-monomer, $w+] 50 A$ UAS285 GFP (Manier, Belote et al., 2010), Cg-gal4 (fat body), da-gal4 (ubiquitous), Tub-gal80ts 286 (ubiquitous) from BDSC (https://bdsc.indiana.edu). Inducible UAS-RNAi lines (Table S1) from 287 VDRC (https://stockcenter.vdrc.at/control/main) (Dietzl, Chen et al., 2007), NIG 288 (https://shigen.nig.ac.jp/fly/nigfly) or previously described (Palm, Sampaio et al., 2012, Parvy et 289 al., 2012)

\section{Mating and related analyses}

292 Mating choice were performed as previously described (Wicker-Thomas et al., 2015). For sperm 293 counting and speed, females were fertilized with $P[w 8$, ProtB-DsRed, $w+]$ males that labelled 294 sperm nuclei. For counting, spermathecae and seminal receptacle were dissected in PBS 1X, 295 fixed 20 min in PFA 4\%, mounted in DABCO and spermatozoa were counted with a Zeiss Imager M2 fluorescent microscope. For sperm speed, seminal receptacle were dissected and mounted in Biggers, Whitten and Whittingham modified medium (95 mM NaCl, $4.8 \mathrm{mM} \mathrm{KCl,} 1.3$ mM CaCl2, 1.2 mM MgSO4, $1.2 \mathrm{mM} \mathrm{KH2PO4,} 5.6 \mathrm{mM}$ glucose, $25 \mathrm{mM} \mathrm{NaHCO}$, 20mM

299 HEPES, $0.6 \%$ fatty acid free BSA, $\mathrm{pH} 7.6$ ), supplemented with $0.5 \mathrm{mM}$ trehalose. Time lapse 300 were imaged with Zeiss Imager M2 fluorescent microscope (10 seconds; 0.15 second frame

301 interval); speed means are from at least 20 spermatozoa per genotype (five females each). For 302 bleach resistance, eggs were collected for $2 \mathrm{hrs}$, incubated with commercial bleach $(3,7 \%$ chlorax) for $10 \mathrm{mn}$, rinsed with water, and numbers of eggs still visible were counted.

304

305 Imaging

306 Dissected oenocytes and ovaries were fixed and labeled with DAPI and Oil-Red-O as previously 307 described (Wicker-Thomas et al., 2015). For sperm flagella immunostaining, eggs were 
308 dechorionated for 2 min in commercial bleach (3,7\% chlorax), fixed in a 1:1 heptane:methanol

309 mixture and stored at $-20^{\circ} \mathrm{C}$. Next, embryos were washed three times for 10 min with PBS $0.1 \%$

310 Triton X100, incubated with primary antibody (anti-acetylated tubulin; Sigma-Aldrich; T6793)

311 and DAPI on a rotating wheel overnight at $4^{\circ} \mathrm{C}$, washed three times (20 min each) and

312 incubated with an anti-mouse antibody Alexa Fluor $568 \mathrm{~nm}$ (Invitrogen; A11061). Samples were

313 mounted in DAPCO and analyzed on a Leica SP8 confocal laser-scanning microscope.

\section{Biochemistry}

316 Experiments performed as previously described: CHC measurement (Wicker-Thomas et al., 317 2015). TAG measurement (Garrido et al., 2015); protein extracts and western-blotting 318 (Montagne, Lecerf et al., 2010). The Smaug antibody was kindly provided by M Simonelig 319 (Chartier, Klein et al., 2015). Quantification of western-blot was performed using ImageJ.

\section{$321 \quad$ Statistics}

322 Statistical analysis were performed using PRISM/Graphpad. Statistical significance were 323 indicated as ${ }^{*},{ }^{* *}$ and ${ }^{* *}$ corresponding to $\mathrm{P}<0.05,0.01$ and 0.001 , respectively. T-test were 324 used for Fig. 1B, 1C, 1D, 2D, 2E, 3A, 3B, 4E, 4F. Chi-2 test were used for Fig. 2C and 4D. 325 ANOVA was used for Table S2.

\section{REFERENCES}

328 Avila FW, Bloch Qazi MC, Rubinstein CD, Wolfner MF (2012) A requirement for the

329 neuromodulators octopamine and tyramine in Drosophila melanogaster female sperm storage.

330 Proc Natl Acad Sci U S A 109: 4562-7

331 Aviles-Pagan EE, Orr-Weaver TL (2018) Activating embryonic development in Drosophila. 
Barber MC, Price NT, Travers MT (2005) Structure and regulation of acetyl-CoA carboxylase genes of metazoa. Biochim Biophys Acta 1733: 1-28

335 Billeter JC, Atallah J, Krupp JJ, Millar JG, Levine JD (2009) Specialized cells tag sexual and species identity in Drosophila melanogaster. Nature 461: 987-91

Chartier A, Klein P, Pierson S, Barbezier N, Gidaro T, Casas F, Carberry S, Dowling P,

338 Maynadier L, Bellec M, Oloko M, Jardel C, Moritz B, Dickson G, Mouly V, Ohlendieck K, Butler-

339 Browne G, Trollet C, Simonelig M (2015) Mitochondrial dysfunction reveals the role of mRNA

340 poly(A) tail regulation in oculopharyngeal muscular dystrophy pathogenesis. PLoS Genet 11:

$341 \mathrm{e} 1005092$

342 Chiang YN, Tan KJ, Chung H, Lavrynenko O, Shevchenko A, Yew JY (2016) Steroid Hormone

343 Signaling Is Essential for Pheromone Production and Oenocyte Survival. PLoS Genet 12:

344 e1006126

345 Chung H, Loehlin DW, Dufour HD, Vaccarro K, Millar JG, Carroll SB (2014) A single gene 346 affects both ecological divergence and mate choice in Drosophila. Science 343: 1148-51

347 Dietzl G, Chen D, Schnorrer F, Su KC, Barinova Y, Fellner M, Gasser B, Kinsey K, Oppel S,

348 Scheiblauer S, Couto A, Marra V, Keleman K, Dickson BJ (2007) A genome-wide transgenic

349 RNAi library for conditional gene inactivation in Drosophila. Nature 448: 151-6

350 Ferveur JF, Savarit F, O'Kane CJ, Sureau G, Greenspan RJ, Jallon JM (1997) Genetic 351 feminization of pheromones and its behavioral consequences in Drosophila males. Science 276: $1555-8$

353 Flybase (2003) The FlyBase database of the Drosophila genome projects and community

354 literature. Nucleic Acids Res 31: 172-5

355 Garrido D, Rubin T, Poidevin M, Maroni B, Le Rouzic A, Parvy JP, Montagne J (2015) Fatty 356 Acid Synthase Cooperates with Glyoxalase 1 to Protect against Sugar Toxicity. PLoS Genet 11: $357 \quad$ e1004995 
Gutierrez E, Wiggins D, Fielding B, Gould AP (2007) Specialized hepatocyte-like cells regulate

359 Drosophila lipid metabolism. Nature 445: 275-80

360 Heifetz Y, Yu J, Wolfner MF (2001) Ovulation triggers activation of Drosophila oocytes. Dev Biol 234: $416-24$

362 Horner VL, Czank A, Jang JK, Singh N, Williams BC, Puro J, Kubli E, Hanes SD, McKim KS,

363 Wolfner MF, Goldberg ML (2006) The Drosophila calcipressin sarah is required for several 364 aspects of egg activation. Curr Biol 16: 1441-6

365 Horner VL, Wolfner MF (2008a) Mechanical stimulation by osmotic and hydrostatic pressure 366 activates Drosophila oocytes in vitro in a calcium-dependent manner. Dev Bio/316: 100-9

367 Horner VL, Wolfner MF (2008b) Transitioning from egg to embryo: triggers and mechanisms of 368 egg activation. Dev Dyn 237: 527-44

369 Jakobsson A, Westerberg R, Jacobsson A (2006) Fatty acid elongases in mammals: their 370 regulation and roles in metabolism. Prog Lipid Res 45: 237-49

371 Kashir J, Nomikos M, Lai FA, Swann K (2014) Sperm-induced Ca2+ release during egg

372 activation in mammals. Biochem Biophys Res Commun 450: 1204-11

373 Krauchunas AR, Wolfner MF (2013) Molecular changes during egg activation. Curr Top Dev 374 Biol 102: 267-92

375 Maier T, Leibundgut M, Boehringer D, Ban N (2010) Structure and function of eukaryotic fatty 376 acid synthases. Q Rev Biophys 43: 373-422

377 Manier MK, Belote JM, Berben KS, Novikov D, Stuart WT, Pitnick S (2010) Resolving 378 mechanisms of competitive fertilization success in Drosophila melanogaster. Science 328: 354$379 \quad 7$

380 Miao YL, Williams CJ (2012) Calcium signaling in mammalian egg activation and embryo 381 development: the influence of subcellular localization. Mol Reprod Dev 79: 742-56 
382 Montagne J, Lecerf C, Parvy JP, Bennion JM, Radimerski T, Ruhf ML, Zilbermann F, Vouilloz N, 383 Stocker H, Hafen E, Kozma SC, Thomas G (2010) The nuclear receptor DHR3 modulates dS6 384 kinase-dependent growth in Drosophila. PLoS Genet 6: e1000937

385 Montagne J., Wicker-Thomas C (2020) Drosophila pheromone production. In Insect Pheromone 386 Biochemistry and Molecular Biology, Blomquist G., Vogt R. G. (eds) pp 163-181. Elsevier 387 Academic Press, London

388 Neubaum DM, Wolfner MF (1999) Wise, winsome, or weird? Mechanisms of sperm storage in 389 female animals. Curr Top Dev Biol 41: 67-97

390 Page AW, Orr-Weaver TL (1997) Activation of the meiotic divisions in Drosophila oocytes. Dev Biol 183: 195-207

392 Palm W, Sampaio JL, Brankatschk M, Carvalho M, Mahmoud A, Shevchenko A, Eaton S (2012) 393 Lipoproteins in Drosophila melanogaster--assembly, function, and influence on tissue lipid 394 composition. PLoS Genet 8: e1002828

395 Parvy JP, Napal L, Rubin T, Poidevin M, Perrin L, Wicker-Thomas C, Montagne J (2012) 396 Drosophila melanogaster Acetyl-CoA-carboxylase sustains a fatty acid-dependent remote signal 397 to waterproof the respiratory system. PLoS Genet 8: e1002925

398 Qiu Y, Tittiger C, Wicker-Thomas C, Le Goff G, Young S, Wajnberg E, Fricaux T, Taquet N, 399 Blomquist GJ, Feyereisen R (2012) An insect-specific P450 oxidative decarbonylase for 400 cuticular hydrocarbon biosynthesis. Proc Natl Acad Sci U S A 109: 14858-63

401 Rekwot PI, Ogwu D, Oyedipe EO, Sekoni VO (2001) The role of pheromones and biostimulation 402 in animal reproduction. Anim Reprod Sci 65: 157-70

403 Sartain CV, Wolfner MF (2013) Calcium and egg activation in Drosophila. Cell Calcium 53: 10-5 404 Swann K, Lai FA (2016) Egg Activation at Fertilization by a Soluble Sperm Protein. Physiol Rev 405 96: 127-49 
406 Tadros W, Goldman AL, Babak T, Menzies F, Vardy L, Orr-Weaver T, Hughes TR, Westwood 407 JT, Smibert CA, Lipshitz HD (2007) SMAUG is a major regulator of maternal mRNA 408 destabilization in Drosophila and its translation is activated by the PAN GU kinase. Dev Cell 12: $409 \quad 143-55$

410 Wicker-Thomas C, Garrido D, Bontonou G, Napal L, Mazuras N, Denis B, Rubin T, Parvy JP, 411 Montagne $\mathrm{J}$ (2015) Flexible origin of hydrocarbon/pheromone precursors in Drosophila 412 melanogaster. J Lipid Res 56: 2094-101

413 Wolfner MF (2011) Precious essences: female secretions promote sperm storage in Drosophila. $414 \quad$ PLoS Biol 9: e1001191

Figure legends

Figure 1: Characterization of an oenocyte FA anabolic pathway required for female fertility: (A) Oenocyte(BO)- or ubiquitous(da)-knockdown of CG6432 induced tracheal flooding in late L2 larvae; in their flooded section, the tracheal trunks (arrows in control: Co) were hardly visible. (B) CHCs amounts in control (black) or 1407>6432-RNAi (green) females $(\mathrm{n}=10)$; 421 Amounts of dienes (di), monoenes (mono), saturated linear (sat), pheromones (HD+ND) and $422 \mathrm{mbCHCs}(\mathrm{mb})$ are listed in Table EV2; note the strong reduction of mbCHCs. (C) TAG content 423 in 0-5 hours prepupae of the following genotypes: Cg-gal4 control (black), Cg>FASN3-RNAi 424 (purple), $C g>C G 6432-R N A i$ (green) and $C g>F A S N 1-R N A i$ (grey). (D) Pupal progeny of promEgal4 females either control (black) or expressing an RNAi to FASN3 (purple), KAR (orange), 426 CG6432 (green), FATP (dark grey), ACC (light grey) or elo ${ }^{C G 6660}$ (white). Five females were mated with five males for two days (0-2d), then, males were removed and females transferred in 428 new tubes every second day $(2-4 d, 4-6 d, 6-8 d)$. Each bar represents the man value of $3-5$ 429 replicates. (E) Oenocyte anabolic pathway producing a VLCFA controlling fertility, where 430 CG6432 and FATP are potential acyl-CoA synthase for the primer used by FASN2/FASN3 and 431 for FA elongation, respectively; enzymes (blue/red) and metabolites (black) are indicated. 
432 Figure 2: Oogenesis and mating: (A-B) Oenocytes (yellow dotted line in A1-3) and stage 9-10

$433\left(^{*}\right)$ and late (\#) egg chambers (B1-3) of promE-gal4 females either control (A1, B1), or directing 434 an RNAi to FASN3 (A2, B2) or CG6432 (A3, B3); tissues were dissected 10-days after mating; 435 lipids and nuclei were labelled with Nile Red (red) and DAPI (silver), respectively; scale bars: 40 $\mu \mathrm{m}(\mathrm{A} 1-3)$ and $100 \mu \mathrm{m}(\mathrm{B} 1-3)$. (C) Mating choice of single wild type males in the presence of two females, one control and one expressing an RNAi to either FASN3 (left) or CG6432 (right); bars represent the percentage of copulation with control (color) or RNAi-expressing (purple or green) females; males tend to prefer CG6432-RNAi females although not significantly. (D-F) eggs (D) and pupae (E) from promE-gal4 females either control (black) or expressing an RNAi to FASN3 (purple) or CG6432 (green); three females were mated with three males for one day, then, males were removed and females transferred in new tubes every day over a 6-day period; index of fertility $(F)$ were evaluated as the ratio of prepupae to eggs.

Figure 3: Sperm activity: (A) Sperm numbers in the spermathecae (left) and seminal receptacle (right) of promE-gal4 females either control (Co) or directing an RNAi to FASN3 or CG6432, one day (black) or five days (grey) after mating $(n>25)$.

(B) Movement speed of spermatozoa in the seminal receptacle of promE-gal4 females either control (black), or directing an RNAi to FASN3 (purple) or CG6432 (green). (C-E) Eggs laid from promE-gal4 fertilized females either control (C), or directing an RNAi to FASN3 (D) or CG6432 (E); eggs were collected for $40 \mathrm{mn}$, nuclei were labeled with DAPI (silver) and sperm flagella (arrows) with an anti-acetylated-tubulin (red); scale bars: $40 \mu \mathrm{m}$.

452 Figure 4; Oocyte activation: (A-C) Eggs laid from promE-gal4 virgin females either control (A), or directing an RNAi to FASN3 (B) or CG6432 (C); eggs were collected for 2 hrs and nuclei were labeled with DAPI (silver); scale bars: $40 \mu \mathrm{m}$. (D) Number of nuclei counted in the egg 455 collections (A-C). (E) Resistance to bleach lysis of eggs laid by promE-gal4 fertilized females 456 either control (black), or directing and RNAi to FASN3 (purple) or CG6432 (green); bars 457 represent means from 27 independent tests, each containing 25 eggs per genotype. (F) 458 Western-blotting to Smaug from protein extracts of dissected ovaries or eggs laid by promE- 
459 gal4 females either virgin or fertilized; ovaries and unfertilized eggs were from control females,

460 fertilized eggs were from females either control or directing an RNAi to FASN3 or CG6432. The 461 graph at the bottom compares the means of four independent blots, where the band intensity of 462 Smaug was normalized to that of the tubulin loading control.

463

464 
Figure 1
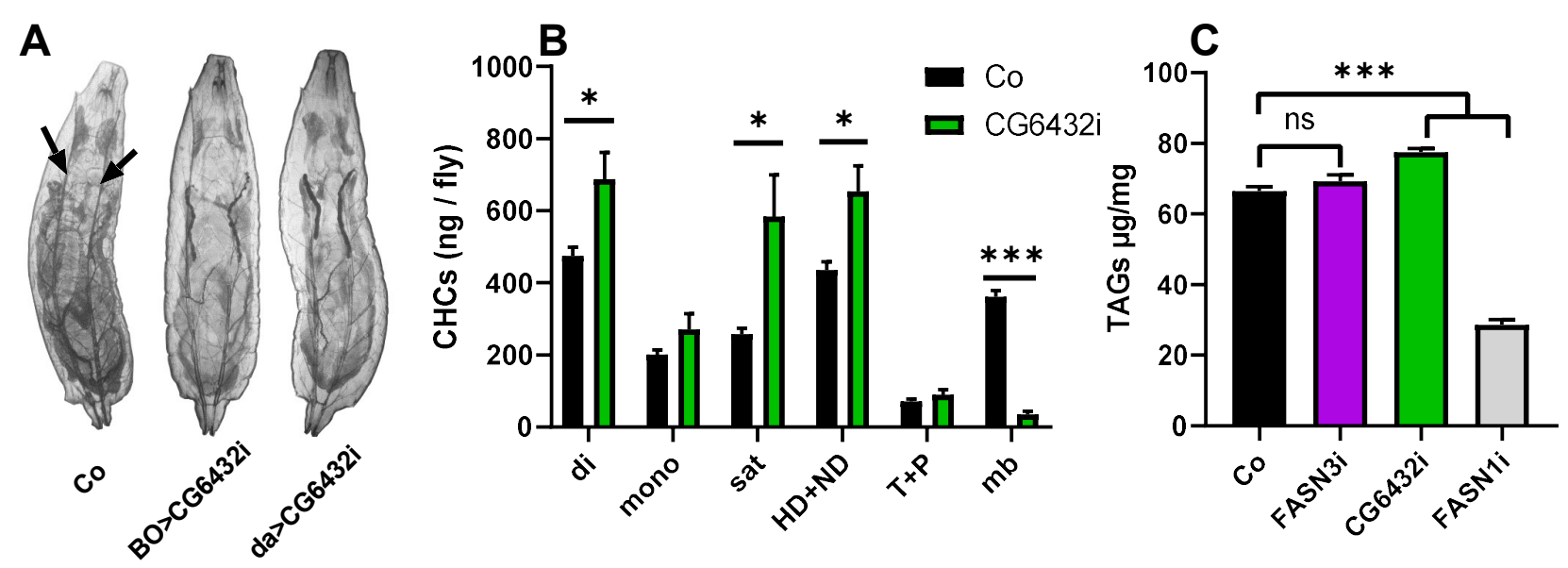

D
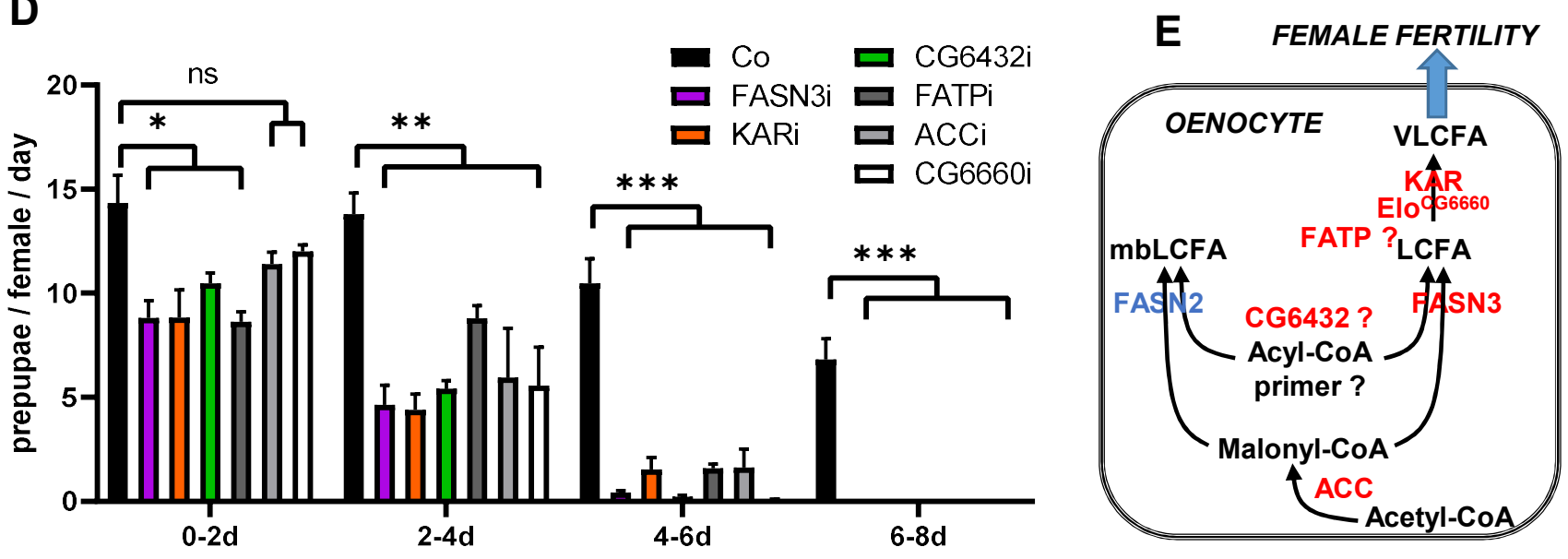
Figure 2
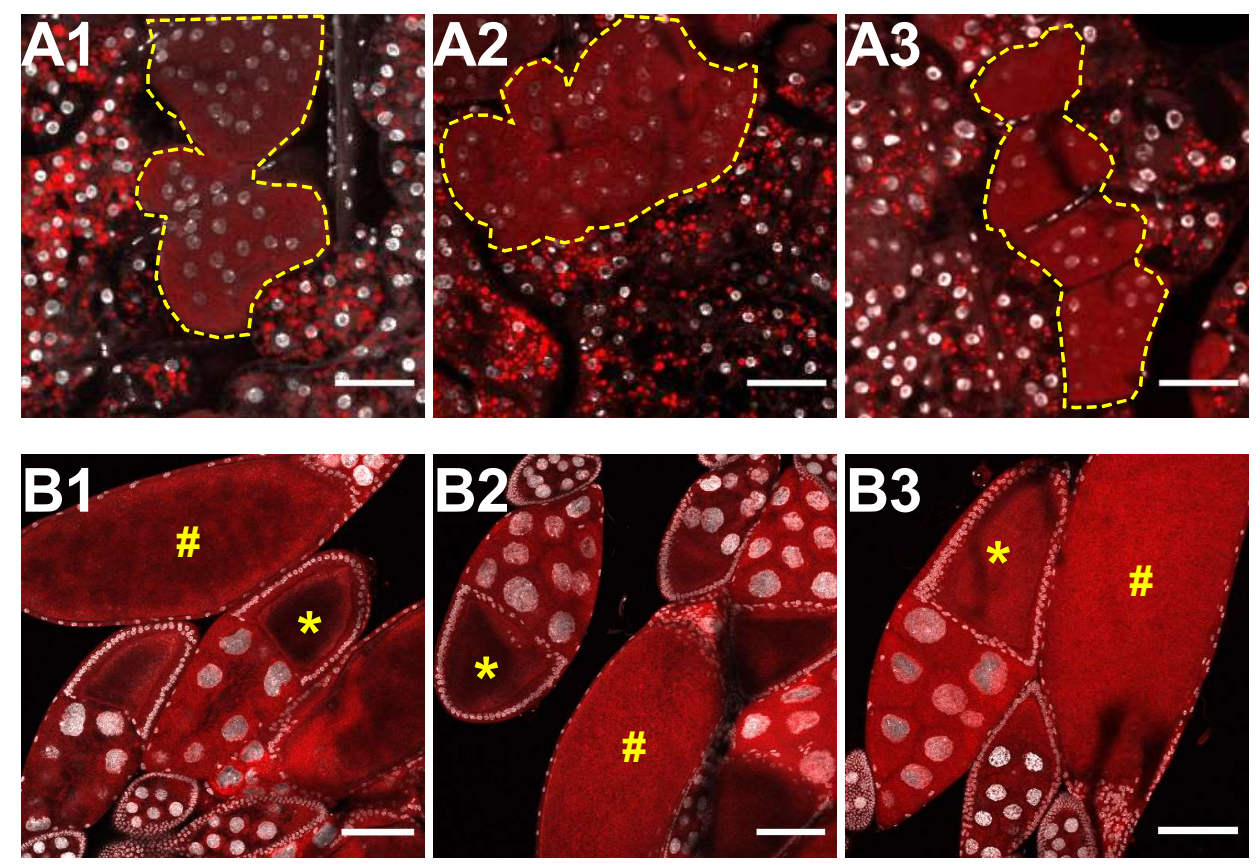

C

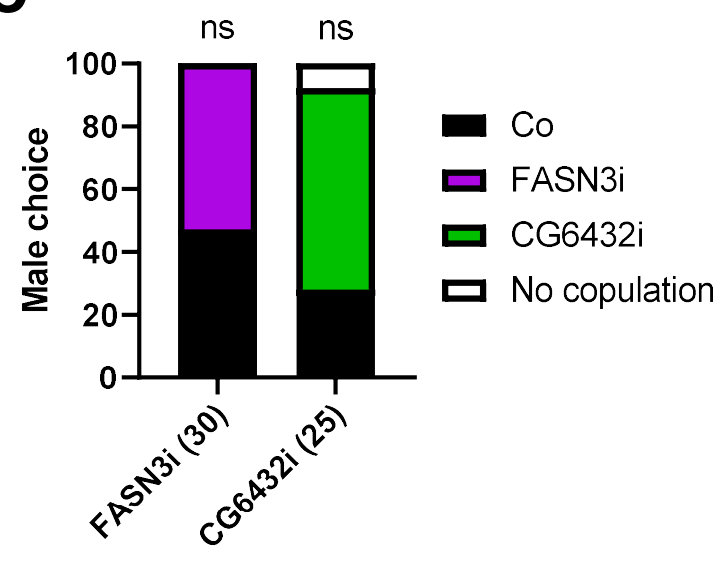

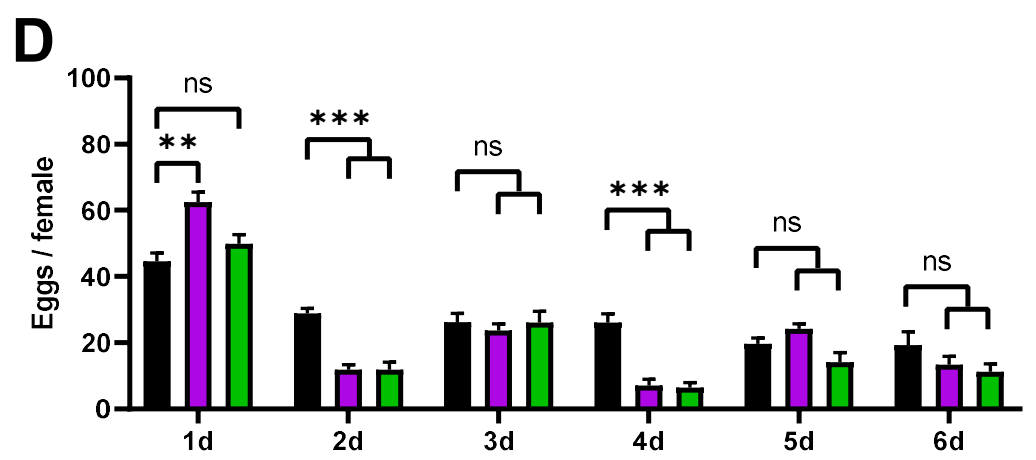

E

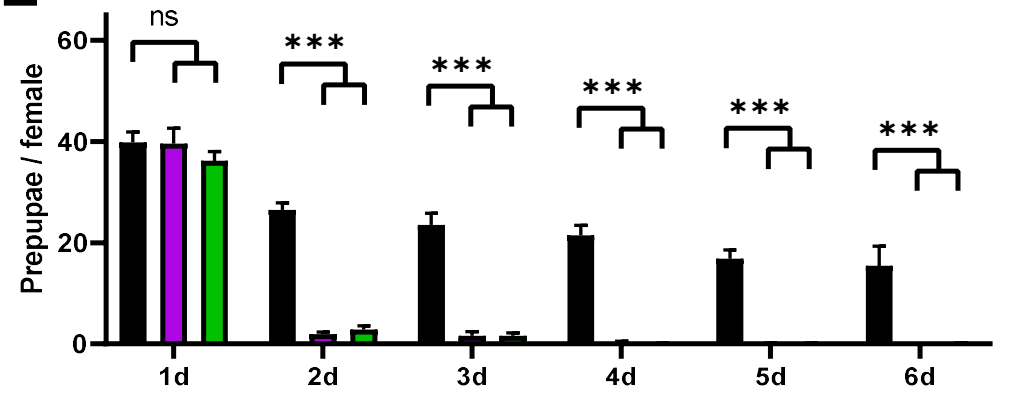

F

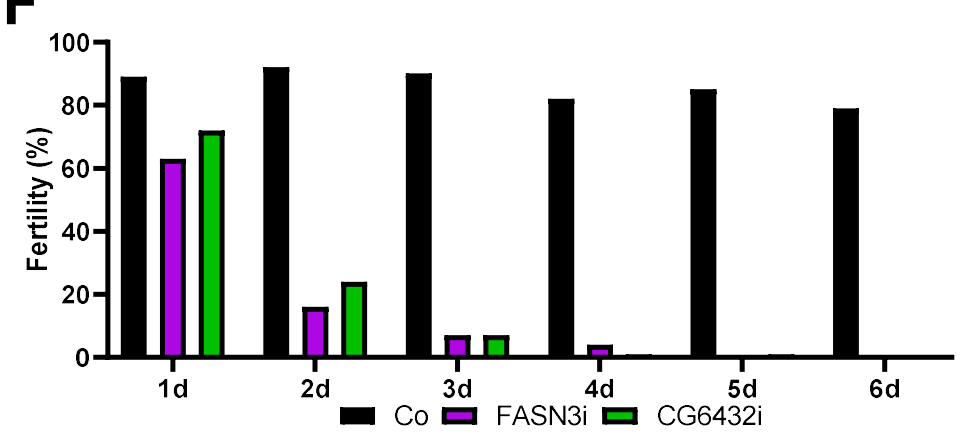


Figure 3
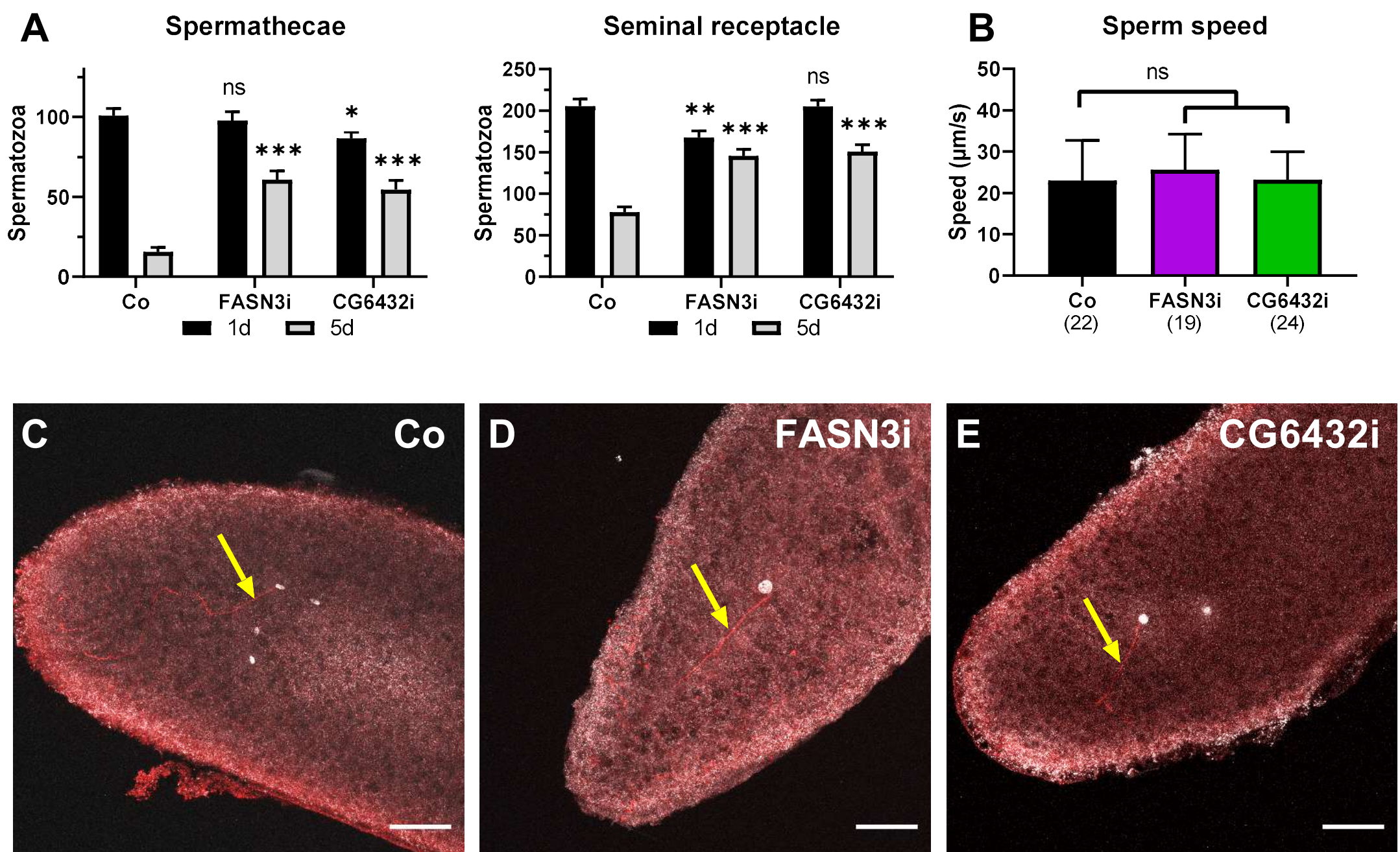
Figure 4

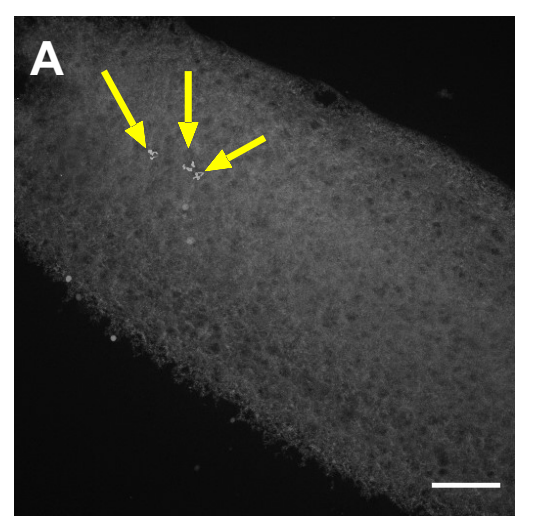

D

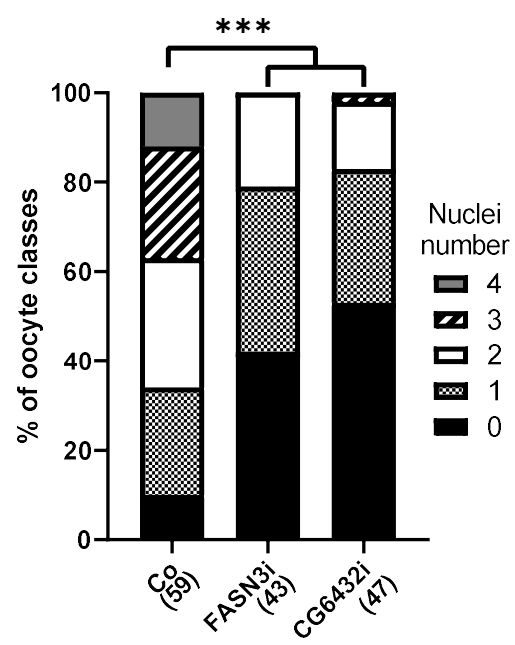

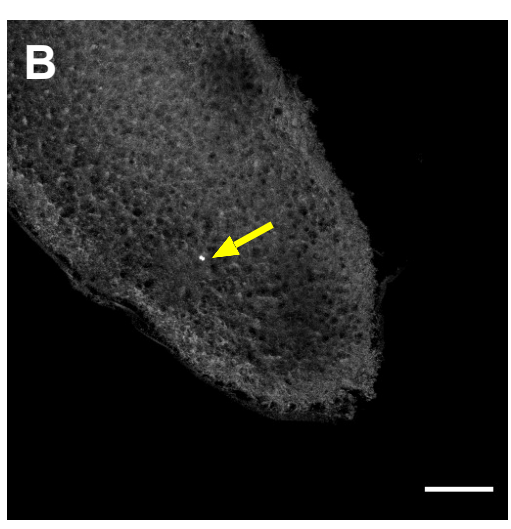

E

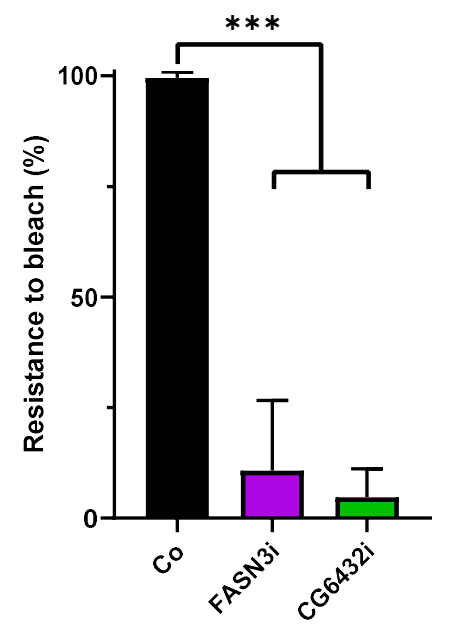

C

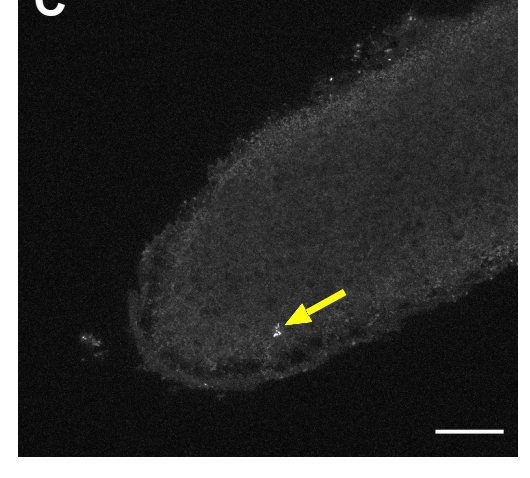

F

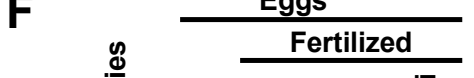

Smg

Tub $\longrightarrow$

具 


\section{A Fatty Acid Anabolic Pathway in Specialized-Cells Remotely Controls Oocyte Activation in Drosophila}

Mickael Poidevin, Nicolas Mazuras, Gwénaëlle Bontonou, Pierre Delamotte, Béatrice Denis, Maëlle Devilliers, Delphine Petit, Claude Wicker-Thomas, and Jacques Montagne

\section{EXTENDED VIEW}

Figure EV1. Screening for sterility.

Figure EV2. Oenocytes structure during lifespan.

Figure EV3. CG6432 blast.

Figure EV4. male CHCs.

Table EV1. List of the genes screened for female sterility

Table EV2. Statistical and $\mathrm{CHC}$ detailed analyses

Movie EV1. Displacement of spermatozoa in the seminal receptacle of promE-gal4 control females.

Movie EV2. Displacement of spermatozoa in the seminal receptacle of promE-gal4>FASN3-Ri.

Movie EV3. Displacement of spermatozoa in the seminal receptacle of promE-gal4>CG6432-Ri. 


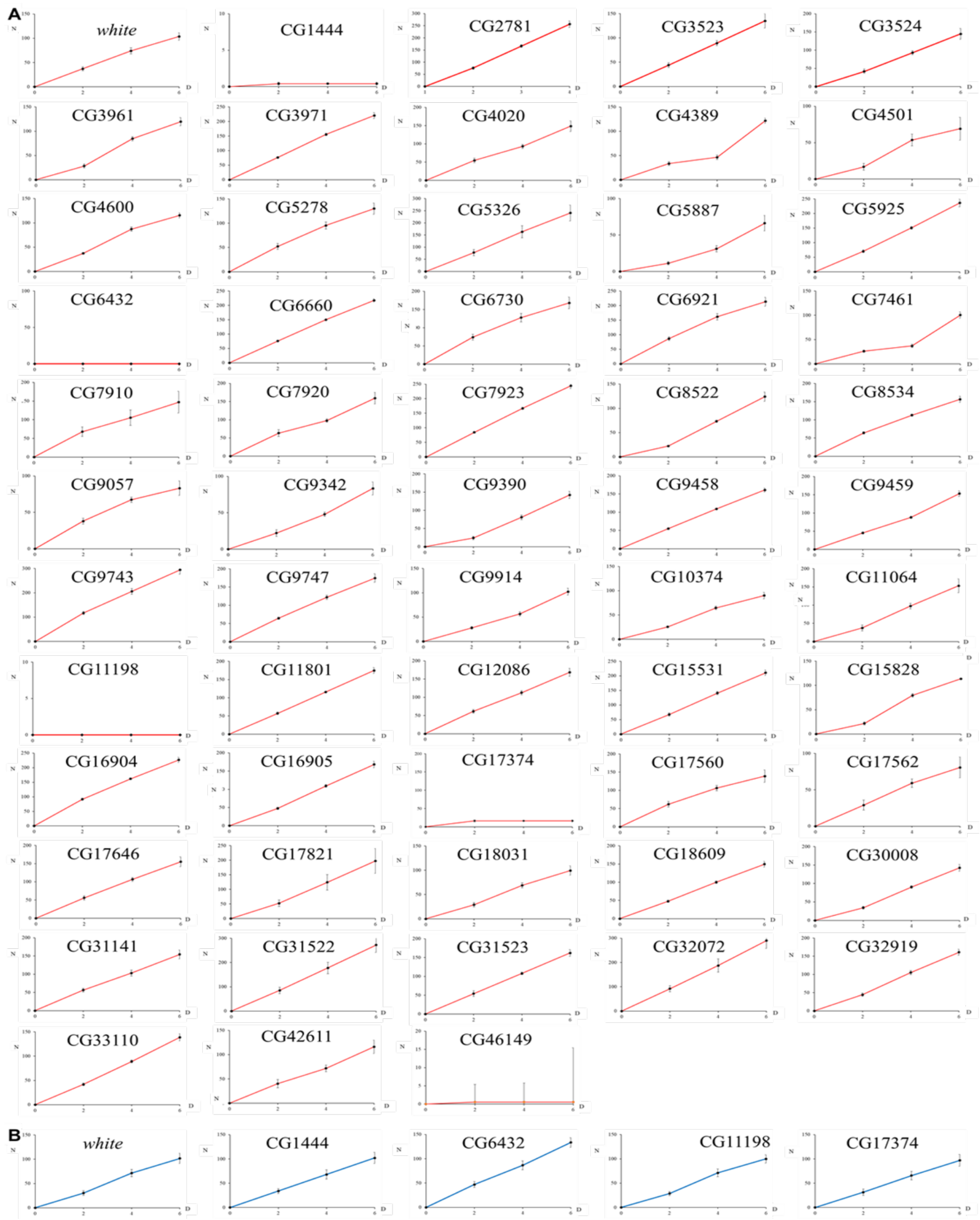

Figure EV1: Screening for sterility. (A) 1407-gal4>UAS-RNAi females crossed to Canto-S males were let to lay eggs during six days $(D)$ in three successive vials and the progeny was counted at adult emergence (N). (B) Reciprocal crosses to test male fertility. 


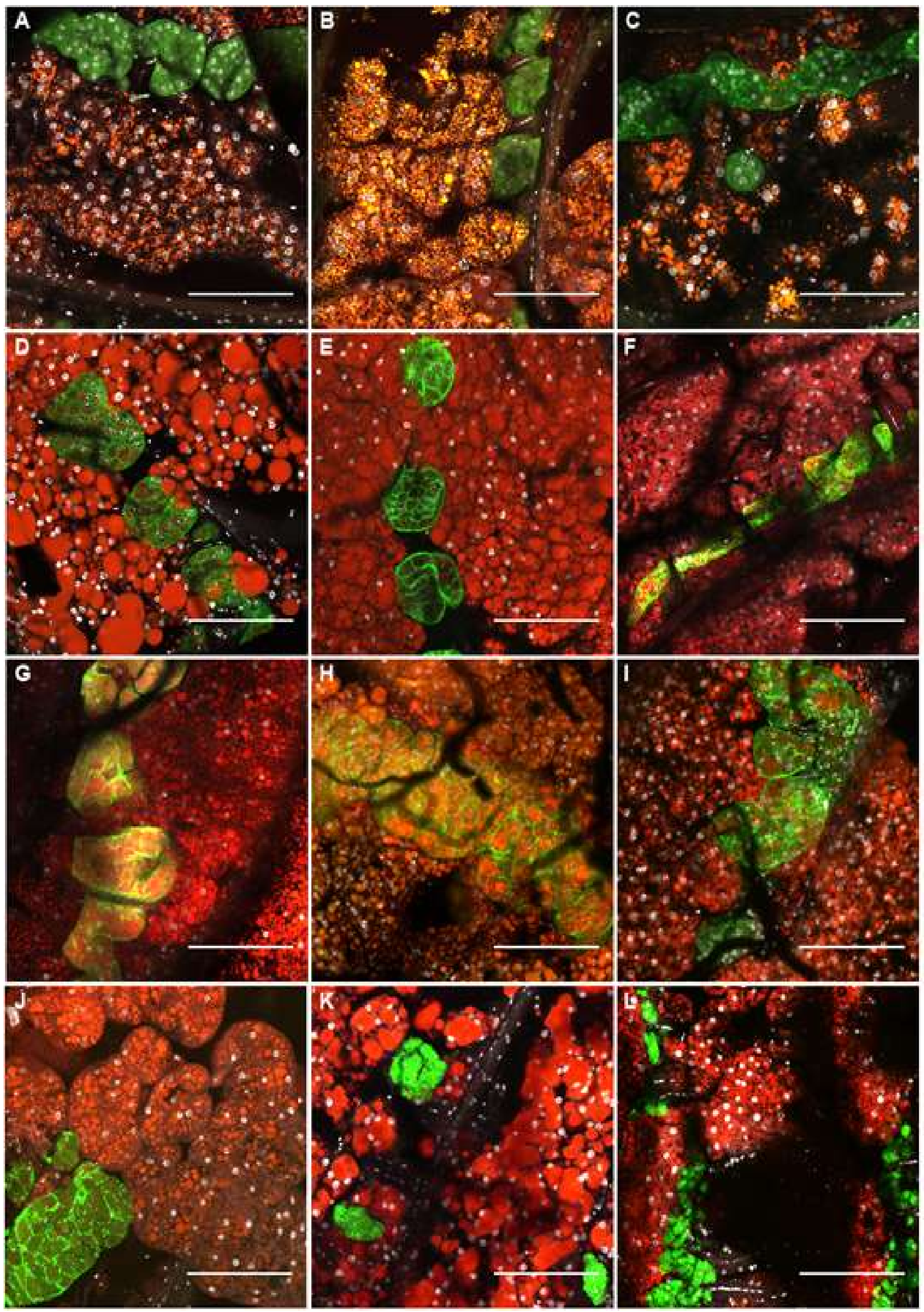



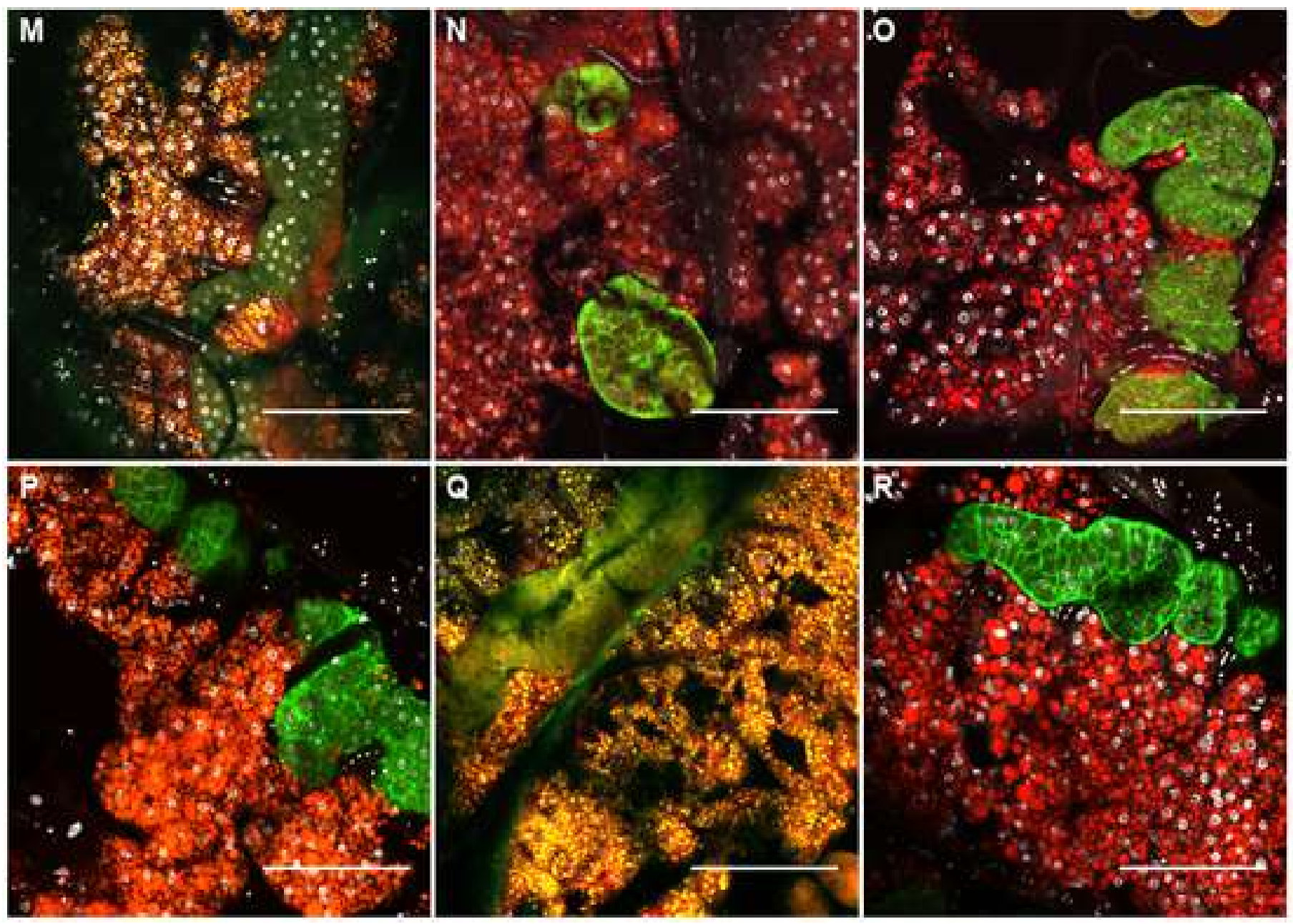

Figure EV2: Oenocytes structure during lifespan. Dorsal abdominal cuticles stained for lipids and nuclei of 1407>UAS-GFP females either control (A-C), or directing UAS-RNAis to KAR (D-F), ACC (G-I), FATP (J-L), FASN3 (M-O) or CG6432 (P-R). Females were dissected 9 days $(A, D, G, J, M, P), 18$ days $(B, E, H, K, N, Q)$ or 27 days $(C, F, O, R)$ after adult emergence, except ACCand FATP-RNAis flies that did not survive longer than 24 days $(I, L)$. Oenocytes were visualized by GFP (green) the nuclei by DAPI (silver) and the fat body by Nile red. Since the Nile red partially interferes with the GFP channel and that the GFP intensity varied a lot for unknown reasons, the lipid staining appeared either red (strong GFP) or orange (low GFP). Note that oenocyte loss appeared earlier in age for FATP-RNAi $(K, L)$ than for KAR-RNAi (F). Scale bars: $100 \mu \mathrm{m}$. 
bioRxiv preprint doi: https://doi.org/10.1101/2021.04.19.440456; this version posted April 20, 2021. The copyright holder for this preprint (which was not certified by peer review) is the author/funder. All rights reserved. No reuse allowed without permission.

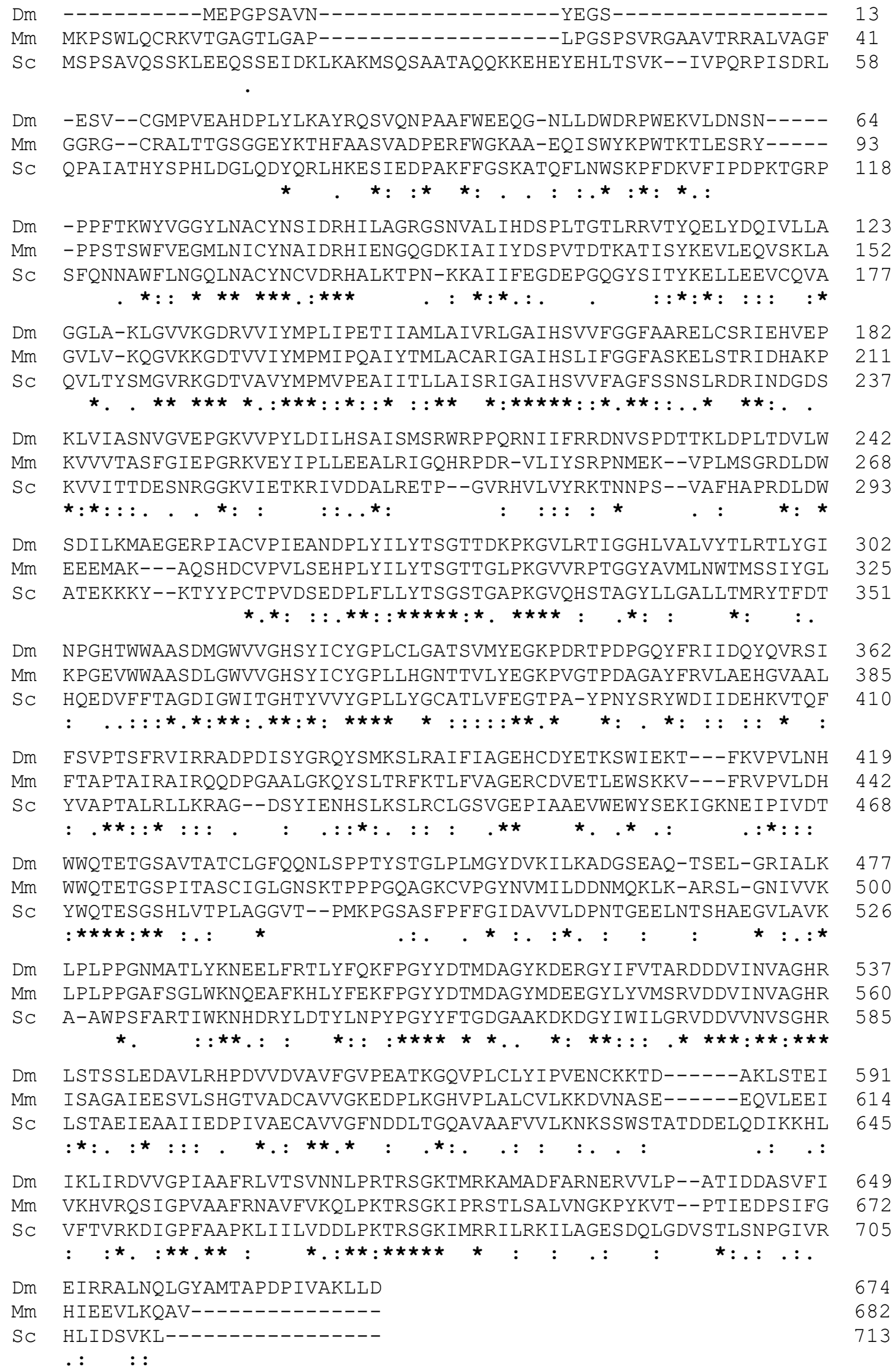

Figure EV3: CG6432 blast. Peptide sequence alignment of CG6432 (Dm) to the best homologues Acss3 in mouse (Mm) and Acs1 in yeast (Sc), using www.uniprot.org. 


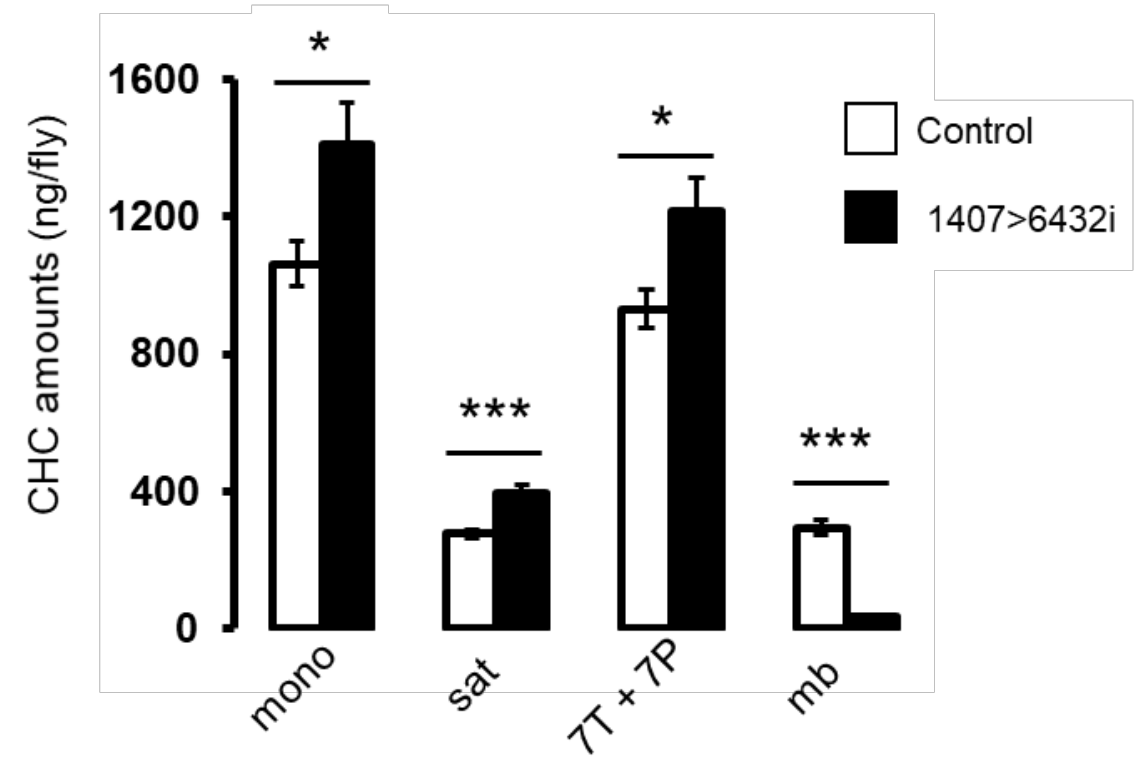

Figure EV4: male CHCs. Means values of CHCs from 10 control (white) or 10 CG6432-RNAi (black) males. 


\begin{tabular}{|c|c|c|c|c|}
\hline CG & Name, Expected function & Line & Stock & Fertility \\
\hline 1444 & Ketoacyl-DH/VLCFA synthesis & 40949 & VDRC & Sterile \\
\hline 2781 & Elongase, VLCFA synthesis & 102543 & VDRC & + \\
\hline 3523 & FASN1, LCFA synthesis & 29349 & VDRC & + \\
\hline 3524 & FASN2, LCFA synthesis & 4290 & VDRC & + \\
\hline 3961 & LCFA-CoA ligase & 37305 & VDRC & + \\
\hline 3971 & Baldspot, Elongase, VLCFA synthesis & 47519 & VDRC & + \\
\hline 4020 & FA-CoA reductase & 107095 & VDRC & + \\
\hline 4389 & Mtpa, b-oxidation & 21845 & VDRC & + \\
\hline 4501 & bgm, LCFA-CoA ligase & 105635 & VDRC & + \\
\hline 4600 & yip2, Thiolase, b-oxidation & 26562 & VDRC & + \\
\hline 5278 & sit, Elongase, VLCFA synthesis & 43091 & VDRC & + \\
\hline 5326 & Elongase, VLCFA synthesis & 47681 & VDRC & + \\
\hline 5887 & desat1, desaturase & 104350 & VDRC & + \\
\hline 5925 & desat2, desaturase & 103666 & VDRC & + \\
\hline 6432 & short chain acyl-CoA ligase & 43451 & VDRC & Sterile \\
\hline 6660 & Elongase, VLCFA synthesis & 101046 & VDRC & + \\
\hline 6660 & Elongase, VLCFA synthesis & 6660R-2 & NIG & ND \\
\hline 6730 & Cyp4d21, monooxygenase & 102401 & VDRC & + \\
\hline 6921 & bond, Elongase, VLCFA synthesis & 102051 & VDRC & + \\
\hline 7461 & Acyl-CoA dehydrogenase, b-oxidation & 7461R-4 & NIG & + \\
\hline 7910 & FA amide hydrolase & 51546 & VDRC & + \\
\hline 7920 & Acetyl-CoA hydrolase & 21577 & VDRC & + \\
\hline 7923 & Fad2, desatF, desaturase & & CWT & + \\
\hline 8522 & SREBP, Transcription factor & 37640 & VDRC & + \\
\hline 8534 & Elongase, VLCFA synthesis & 107515 & VDRC & + \\
\hline 9057 & Lsd-2, Lipid storage & 40734 & VDRC & + \\
\hline 9342 & Mtp, Lipid transport & 110414 & VDRC & + \\
\hline 9390 & AcCoAS, Acetate-CoA ligase & 100281 & VDRC & + \\
\hline 9458 & Elongase, VLCFA synthesis & 48702 & VDRC & + \\
\hline 9459 & Elongase, VLCFA synthesis & 48905 & VDRC & + \\
\hline 9743 & FA desaturase & 108185 & VDRC & + \\
\hline 9747 & FA desaturase & 1394 & VDRC & + \\
\hline 9914 & 3-hydroxyacyl-CoA dehydrogenase & 106649 & VDRC & + \\
\hline 10374 & Lsd-1, Lipid storage & 30844 & VDRC & + \\
\hline 11064 & apolpp, Lipid transport & 100944 & VDRC & + \\
\hline 11198 & ACC, malonyl-CoA synthesis & 8105 & VDRC & Sterile \\
\hline 11801 & Elo68 $\beta$, Elongase, VLCFA synthesis & 103506 & VDRC & + \\
\hline 12086 & cue, LDL receptor & 104645 & VDRC & + \\
\hline 15531 & FA desaturase & 1397 & VDRC & + \\
\hline 15828 & Apoltp, Lipid transport & & S Eaton & + \\
\hline 16904 & Elongase, VLCFA synthesis & 106515 & VDRC & + \\
\hline 16905 & eloF, VLCFA synthesis & 16905-R1 & NIG & + \\
\hline 17374 & FASN3, LCFA synthesis & & JM & Sterile \\
\hline 17560 & FA-CoA reductase & 104756 & VDRC & + \\
\hline 17562 & FA-CoA reductase & 37365 & VDRC & + \\
\hline 17646 & $A B C$ transporter-like & 100378 & VDRC & + \\
\hline 17821 & Elongase, VLCFA synthesis & 4997 & VDRC & + \\
\hline 18031 & FarO, FA-CoA reductase & 30220 & VDRC & + \\
\hline 18609 & Elongase, VLCFA synthesis & 4994 & VDRC & + \\
\hline 30008 & Elongase, VLCFA synthesis & 6760 & VDRC & + \\
\hline 31141 & Elongase, VLCFA synthesis & 100460 & VDRC & + \\
\hline 31522 & Elongase, VLCFA synthesis & 106652 & VDRC & + \\
\hline 31523 & Elongase, VLCFA synthesis & 45226 & VDRC & + \\
\hline 32072 & elo68 $\alpha$, Elongase, VLCFA synthesis & 9206 & VDRC & + \\
\hline 32919 & Fatty acyl-CoA reductase & 6090 & VDRC & + \\
\hline 33110 & Elongase, VLCFA synthesis & 6926-R2 & NIG & + \\
\hline 42611 & mgl, LDL receptor & 105071 & VDRC & + \\
\hline 46149 & Fatp, lipid transport, FA-CoA ligase & 9406 & VDRC & Sterile \\
\hline
\end{tabular}

Table EV1. List of the genes screened for female sterility (fertility column) using the 1407-gal4 driver. RNAi lines were provided by NIG or VDRC; three of them have been generated in S Eaton, CWT or JM laboratories (Stock column). UAS-RNAi lines that produced a sterile phenotype were reused with the promE-gal4 driver, except 6660R-2 used to knockdown elo ${ }^{\text {CG6660. }}$. 


\begin{tabular}{cccc}
\hline Males CHC & $\boldsymbol{P}$ & $\mathbf{6 4 3 2 i}-\boldsymbol{C}$ & $\mathbf{6 4 3 2 i}$ \\
\hline Tot & 0.24 & $1634.1 \pm 91.8$ & $1840.3 \pm 146.8$ \\
\hline $9-\mathrm{T}$ & $\mathbf{0 . 0 0 3}$ & $2.50 \pm 0.25$ & $3.78 \pm 0.28$ \\
$7-\mathrm{T}$ & $\mathbf{0 . 0 0 2}$ & $51.33 \pm 1.05 .2$ & $55.7 \pm 0.61$ \\
$5-\mathrm{T}$ & 0.25 & $3.72 \pm 0.18$ & $4.14 \pm 0.31$ \\
$23: 0$ & $\mathbf{0 . 0 0 4}$ & $13.54 \pm 0.41$ & $14.69 \pm 0.30$ \\
$\mathrm{Me}-24$ & $<.001$ & $3.03 \pm 0.24$ & $0.38 \pm 0.06$ \\
$9-\mathrm{P}$ & $\mathbf{0 . 0 0 3}$ & $1.27 \pm 0.14$ & $2.24 \pm 0.14$ \\
$7-\mathrm{P}$ & $<.001$ & $5.62 \pm 0.19$ & $10.24 \pm 0.53$ \\
$5-\mathrm{P}$ & 0.13 & $0.12 \pm 0.07$ & $0.34 \pm 0.11$ \\
$25: 0$ & 0.50 & $1.85 \pm 0.19$ & $2.05 \pm 0.22$ \\
$\mathrm{Me}-26$ & $<.001$ & $6.68 \pm 0.43$ & $0.42 \pm 0.11$ \\
$27: 0$ & $<.001$ & $1.20 \pm 0.15$ & $2.37 \pm 0.21$ \\
$\mathrm{Me}-28$ & $<.001$ & $7.76 \pm 0.61$ & $0.62 \pm 0.10$ \\
$29: 0$ & $<.001$ & $0.47 \pm 0.11$ & $2.66 \pm 0.24$ \\
\hline
\end{tabular}

\begin{tabular}{cccc}
\hline Females CHC & $\boldsymbol{P}$ & $\mathbf{6 4 3 2 i} \boldsymbol{i}$ & $\mathbf{6 4 3 2 i}$ \\
\hline Tot $23-29$ & 0.26 & $1291.6 \pm 64.7$ & $1574.7 \pm 236.1$ \\
\hline $9-\mathrm{T}$ & 0.25 & $0.70 \pm 0.11$ & $0.89 \pm 0.11$ \\
$7-\mathrm{T}$ & 0.75 & $3.32 \pm 0.38$ & $3.47 \pm 0.29$ \\
$5-\mathrm{T}$ & $\mathbf{0 . 0 3}$ & $0.26 . \pm 0.06$ & $0.60 \pm 0.13$ \\
$23: 0$ & 0.05 & $11.22 \pm 0.62$ & $13.39 \pm 0.84$ \\
$7,11-\mathrm{PD}$ & $\mathbf{0 . 0 4}$ & $2.44 \pm 0.30$ & $1.68 \pm 0.16$ \\
Me-24 & $<.001$ & $1.88 \pm 0.23$ & $0.09 \pm 0.01$ \\
$9-\mathrm{P}$ & 1 & $3.14 \pm 0.31$ & $3.14 \pm 0.37$ \\
$7-\mathrm{P}$ & 0.96 & $2.07 \pm 0.18$ & $2.06 \pm 0.19$ \\
$5-\mathrm{P}$ & 0.42 & $0.16 \pm 0.06$ & $0.23 \pm 0.06$ \\
$25: 0$ & 0.29 & $5.24 \pm 0.28$ & $5.83 \pm 0.48$ \\
$7,11-\mathrm{HD}$ & $<.001$ & $18.84 \pm 0.72$ & $13.84 \pm 0.56$ \\
Me-26 & $<.001$ & $16.37 \pm 0.57$ & $0.60 \pm 0.14$ \\
$9-\mathrm{H}$ & 0.05 & $3.12 \pm 0.14$ & $2.56 \pm 0.23$ \\
$7-\mathrm{H}$ & $\mathbf{0 . 0 1}$ & $2.20 \pm 0.16$ & $2.90 \pm 0.18$ \\
$5-\mathrm{H}$ & $\mathbf{0 . 0 1}$ & $0.05 \pm 0.02$ & $0.19 \pm 0.05$ \\
$27: 0$ & $<.001$ & $2.78 \pm 0.23$ & $10.17 \pm 0.44$ \\
$7,11-\mathrm{ND}$ & $<.001$ & $14.82 \pm 0.52$ & $28.95 \pm 1.55$ \\
Me-28 & $<.001$ & $9.23 \pm 0.42$ & $1.37 \pm 0.34$ \\
$9-\mathrm{N}$ & 0.11 & $0.19 \pm 0.07$ & $0.40 \pm 0.10$ \\
$7-\mathrm{N}$ & 0.06 & $0.22 \pm 0.08$ & $0.44 \pm 0.08$ \\
$29: 0$ & $<.001$ & $0.65 \pm 0.07$ & $6.50 \pm 0.55$ \\
\hline
\end{tabular}

Table EV2. Statistical and CHC detailed analyses: Analysis of 1407>CG6432-Ri (6432i) 4-5-day old males (top) or females (bottom). First column: CHC identities; elemental composition is indicated as the carbon chain length followed by the number of double bonds; Me- are mbCHCs. CHCs are expressed in $\mathrm{ng} /$ fly (Tot) or in percentages relative to total $\mathrm{CHC}$ amount as the mean $( \pm$ SEM) of $\mathrm{CHCs}$ produced by 10 flies kept for 4 days at $25^{\circ} \mathrm{C}$. 